\title{
Independent Collections of Translates of Boxes and a Conjecture due to Grünbaum*
}

\author{
Daniel Q. Naiman ${ }^{1}$ and Henry P. Wynn ${ }^{2}$ \\ ${ }^{1}$ Department of Mathematical Sciences, The Johns Hopkins University, \\ Baltimore, MD 21218, USA \\ dan@jesse.mts.jhu.edu \\ ${ }^{2}$ City University, London, England \\ sc310@city.ac.uk
}

\begin{abstract}
A collection of $n$ sets $A_{1}, \ldots, A_{n}$ is said to be independent provided every set of the form $X_{1} \cap \cdots \cap X_{n}$ is nonempty, where each $X_{i}$ is either $A_{i}$ or $A_{i}^{c}$. We give a simple characterization for when translates of a given box form an independent set in $R^{d}$. We use this to show that the largest number of such boxes forming an independent set in $R^{d}$ is given by $\lfloor 3 d / 2\rfloor$ for $d \geq 2$. This settles in the negative a conjecture of Grünbaum (1975), which states that the maximum size of an independent collection of sets homothetic to a fixed convex set $C$ in $R^{d}$ is $d+1$. It also shows that the bound of $2 d$ of Rényi $e t$ al. (1951) for the maximum number of boxes (not necessarily translates of a given one) with sides parallel to the coordinate axes in an independent collection in $R^{d}$ can be improved for these special collections.
\end{abstract}

\section{Introduction}

A finite collection of sets $\mathscr{A}=\left\{A_{i}, i=1, \ldots, n\right\}$ is said to be independent if $\bigcap_{i=1}^{n} X_{i} \neq \varnothing$, whenever $X_{i}=A_{i}$ or $A_{i}^{c}$ for $i=1, \ldots, n$.

Marczewski (1947) describes how the notion of independence is related to the problem of extending measures. Indeed, is independent if and only if the every choice of $P\left(A_{i}\right) \in[0,1], i=1, \ldots, n$, there exists a unique probability measure $P$ defined on the algebra of sets generated by $\mathscr{A}$ under which $A_{1}, \ldots, A_{n}$ are (probabilistically) independent.

Use of independence leads to simplifications to the classical inclusion-exclusion identity. In fact, Naiman and Wynn (1991b) show for any collection of sets that if the maximum size of an independent subcollection is $k$, then the indicator

\footnotetext{
* Daniel Q. Naiman was supported by National Science Foundation Grant No. DMS-9103126. Henry P. Wynn was supported by the Science and Engineering Research Council, UK.
} 
function of any set in the algebra generated by the collection can be expressed as a linear combination of indicator functions of intersections of at most $k$ of the sets. Naiman and Wynn (1991a) refer to such a formula as a generalized inclusion-exclusion formula of depth $k$, and demonstrate how the Voronoi decomposition can be used to find depth $d+1$ formulas for indicator functions of unions of balls in $R^{d}$. This has led to speculation as to whether a similar simplification holds for other types of sets. A consequence of Theorem 1 below is that there is a depth $\lfloor 3 d / 2\rfloor$ formula for the indicator function of any set in the algebra generated by the collection of boxes in $R^{d}$, all of which are translates of a given box.

Rényi et al. (1951) show the maximum number of independent boxes (with sides parallel to the coordinate axes) in $R^{d}$ is $2 d$, the maximum number of independent balls of equal radius in $R^{d}$ is $d+1$, and if $n(k)$ denotes the maximum number of independent $k$-gons in the plane, then $\lim _{k \rightarrow \infty}\left(n(k) / \log _{2}(k)\right)=1$. Grünbaum (1975) shows that their proof of the third statement is incorrect by giving a counterexample to a condition they claim holds, and he provides a correct proof. Based on the results of Rényi et al. he conjectures that the maximum number of independent sets in $R^{d}$ homothetic to a given compact convex set $C$ is $d+1$.

The term box in $R^{d}$ is used for a set of the form $I_{1} \times \cdots \times I_{d}$, where each $I_{j}$ is a finite one-dimensional interval, and the intervals are in linearly independent subspaces. All intervals below are assumed to be closed. For any dimension $d$ we let $M(d)$ denote the maximum size of an independent family of sets in $R^{d}$ consisting of boxes, all of which are translates of a given one. Since a linear transformation can be applied, it may be assumed that the boxes in any such collection are unit cubes, that is, sets of the form $x+[0,1]^{d}$. Futhermore, a straightforward argument shows that we may restrict attention to collections of cubes in general position, meaning that the intervals obtained by projecting the cubes to any given coordinate axis have distinct endpoints.

We give a simple characterization (Theorem 2) of when collections of unit cubes in general position form an independent family, and we use this to prove the following result:

Theorem 1. The maximum number of boxes in an independent collection in $R^{d}$, all of which are translates of one another, is given by $M(d)=\lfloor 3 d / 2\rfloor$ for $d \geq 2$.

Thus, we verify Grünbaum's conjecture is false for $d \geq 4$ even for this very special class of sets. Furthermore, we improve on the upper bound of Rényi et al. (1951) for this special case.

\section{Preliminaries}

Definition 1. The diagram of an indexed collection of sets $A_{i}, i \in I$, is the collection of index sets $J$ for which

$$
\bigcap_{i \in J} A_{i} \cap \bigcap_{i \notin J} A_{i}^{c} \neq \varnothing
$$

Thus, $N$ sets are independent if and only if the cardinality of their diagram is $2^{N}$. 
Remark 1. If $A_{1}, \ldots, A_{N}$ is a collection of boxes in $R^{d}$ having diagram $\mathscr{D}$, and if $I_{j}^{(r)}$ denotes the projection of $A_{j}$ onto the $r$ th coordinate axis, then a simple argument shows that $\mathscr{D}$ consists of all index sets of the form $\bigcap_{r=1}^{d} J^{(r)}$ where $J^{(r)} \in \mathscr{D}(r), r=1, \ldots, d$, where $\mathscr{D}(r)$ denotes the diagram corresponding to the collection $I_{1}^{(r)}, \ldots, I_{N}^{(r)}$.

Definition 2. A sequence $m_{1}, m_{2}, \ldots, m_{2 N}$, where each integer in $\{1, \ldots, N\}$ appears exactly twice, is said to be an admissible sequence of length $2 N$ if it contains no subsequence of the form ijii.

Remark 2. Given unit intervals $I_{1}, \ldots, I_{N}$ in general position in the real line, the sequence of indices obtained by ordering the endpoints of the intervals in increasing order, and replacing the endpoints by their indices, is easily seen to be admissible. It is equally easy to verify that every admissible sequence arises in this manner.

Lemma 1. Let $I_{j}, j=1, \ldots, N$, be a collection of unit intervals in general position whose diagram is $\mathscr{D}$. Then $\{1, \ldots, N\} \in \mathscr{D}$ if and only there is a permutation $\pi_{1}, \ldots, \pi_{N}$ for which the associated admissible sequence is given by

$$
\pi_{1}, \ldots, \pi_{N}, \pi_{1}, \ldots, \pi_{N}
$$

Proof. This is an immediate consequence of Helly's theorem in one dimension.

Definition 3. A ray of a permutation $\pi_{1}, \ldots, \pi_{N}$ is a subset of $\{1, \ldots, N\}$ which is either of the form $\left\{\pi_{i}: i \leq k\right\}$ or of the form $\left\{\pi_{i}: i \geq k\right\}$ for some $k \in\{0, \ldots, N+1\}$.

Note that $\varnothing$ and $\{1, \ldots, N\}$ are rays since we allow $k=0$ and $k=N+1$. Also, the complement of a ray is a ray.

The following result is elementary.

Lemma 2. Suppose $I_{j}, j=1, \ldots, N$, is a collection of unit intervals in general position with $\{1, \ldots, N\} \in \mathscr{D}$, the diagram of the collection. Then $J \in \mathscr{D}$ if and only if $J$ is a ray of $\pi_{1}, \ldots, \pi_{N}$, the permutation obtained from the associated admissible sequence.

\section{Combinatorial Characterization and a Proof of Theorem 1}

Now we characterize when an independent family of unit cubes (in general position) of a given size exists. 
Theorem 2. There exists an independent collection of $N$ unit cubes in general position in $R^{d}$ if and only if there exist d permutations $\pi^{(r)}=\pi_{1}^{(r)}, \ldots, \pi_{N}^{(r)}, r=1, \ldots, d$, such that every $J \subseteq\{1, \ldots, N\}$ can be expressed as $\bigcup_{r=1}^{d} J^{(r)}$, where each $J^{(r)}$ is a ray of $\pi^{(r)}$.

When such permutations exist, it is easy to construct an independent collection of cubes for which $\pi^{(r)}$ gives the ordering of the intervals obtained by projecting the cubes onto the $r$ th coordinate axis.

Proof. First suppose $A_{i}, i=1, \ldots, N$, is an independent collection of unit cubes in general position in $R^{d}$, and let $I_{i}^{(r)}$ denote the unit interval obtained by projecting $A_{i}$ onto the $r$ th coordinate axis. Since $\bigcap_{i=1}^{N} A_{i} \neq \varnothing$ we have $\bigcap_{i=1}^{N} I_{i}^{(r)} \neq \varnothing$ for $r=1, \ldots, d$. By Lemma 1 , the admissible sequence associated with the collection $I_{i}^{(r)}, i=1, \ldots, N$, is of the form (1) for some permutation $\pi^{(r)}=\pi_{1}^{(r)}, \ldots, \pi_{N}^{(r)}$.

For any $J \subseteq\{1, \ldots, N\}$ independence of the cubes guarantees that $J^{c} \in \mathscr{D}$. Let $\mathscr{D}(r)$ denote the diagram of the collection $I_{i}^{(r)}, i=1, \ldots, N$. Using Remark 1 $J^{c}=\bigcap_{r=1}^{d} J^{(r)}$, where each $J^{(r)} \in \mathscr{D}(r)$, and, by Lemma 2 , each $J^{(r)}$ is a ray of $\pi^{(r)}$. Since the complement of a ray is also a ray, $J$ is a union of rays of the $\pi^{(r)}$.

Conversely, suppose we can find permutations $\pi^{(r)}, r=1, \ldots, d$, with the stated property. For each $r$, the sequence (1) with $\pi=\pi^{(r)}$ is admissible, so we can apply Remark 2 to conclude that this is the associated admissible sequence for some unit intervals $I_{1}^{(r)}, \ldots, I_{N}^{(r)}$, in general position. Applying Lemma 1 gives $\{1, \ldots, N\} \in \mathscr{D}(r)$, the diagram for $I_{1}^{(r)}, \ldots, I_{N}^{(r)}$.

Now define unit cubes $A_{i}=I_{i}^{(1)} \times \cdots \times I_{i}^{(d)}$, for $i=1, \ldots, N$, and let $\mathscr{D}$ denote the diagram for the collection of cubes. For any $J \subseteq\{1, \ldots, N\}$ put $J^{c}=\bigcup_{r=1}^{d} J^{(r)}$, where each $J^{(r)}$ is a ray of $\pi^{(r)}$. It follows that $J=\bigcap_{r=1}^{d} J^{(r) c}$, and each $J^{(r) c}$ is a ray of $\pi^{(r)}$, so Lemma 2 gives $J^{(r) c} \in \mathscr{D}(r)$. By Remark $1 J \in \mathscr{D}$, so the cubes are independent.

Proof of Theorem 1. We prove this result in two steps. First we show the function $M(d)$ defined above is an upper bound for the number of elements in an independent collection of cubes $A_{1}, \ldots, A_{N}$ in general position in $R^{d}$. For such a collection use the permutations $\pi^{(1)}, \ldots, \pi^{(d)}$ given in Theorem 2 to define twoelement sets $T^{(r)}=\left\{\pi_{1}^{(r)}, \pi_{N}^{(r)}\right\}$ for $r=1, \ldots, d$. Theorem 2 guarantees that each singleton $\{j\}$, for $j=1, \ldots, N$, is a union of rays (some possibly empty) of the $d$ permutations, so that $\bigcup_{r=1}^{d} T^{(r)}=\{1, \ldots, N\}$. Furthermore, each $T^{(r)}=\left\{\pi_{1}^{(r)}, \pi_{N}^{(r)}\right\}$ must itself be a union of rays. Since $N>2$ this implies some distinct $T^{(s)}$ contains either $\pi_{1}^{(r)}$ or $\pi_{N}^{(r)}$. Thus, for any $r$ there exists $s \neq r$ such that $T^{(r)} \cap T^{(s)} \neq \varnothing$

In graph-theoretic terms, the sets $T^{(r)}$ define a graph with vertices $1, \ldots, N$ and $d$ edges, one for each $T^{(r)}$ connecting its two elements, and this graph has the property that each edge has a vertex in common with some other edge. It is an elementary exercise to check that the number $N$ of vertices in such a graph satisfies $N \leq\lfloor 3 d / 2\rfloor$.

Now suppose $d$ is a positive integer and take $N=\lfloor 3 d / 2\rfloor$. If $d$ is even there 
exist $d$ permutations of $\{1, \ldots, N\}$ whose elements can be constrained as follows:

$\begin{array}{ccccc}1 & \cdots & \cdots & . & 2 \\ 1 & \cdots & \cdots & 2 & 3 \\ 4 & \cdots & \cdots & \cdot & 5 \\ 4 & \cdots & \cdots & 5 & 6 \\ 7 & \cdots & \cdots & \cdot & 8 \\ 7 & \cdots & \cdots & 8 & 9 \\ \vdots & \vdots & \vdots & \vdots & \vdots \\ N-5 & \cdots & \cdots & \cdot & N-4 \\ N-5 & \cdots & \cdots & N-4 & N-3 \\ N-2 & \cdots & \cdots & \cdot & N-1 \\ N-2 & \cdots & \cdots & N-1 & N\end{array}$

Every subset of each of the sets $\{1,2,3\},\{4,5,6\}, \ldots,\{N-2, N-2, N\}$ is a union of rays, so every $J \subseteq\{1, \ldots, N\}$ is a union of rays.

When $d$ is odd take $d$ permutations of $\{1, \ldots, N\}$ as follows:

$\begin{array}{cccc}1 & \cdots & . & 2 \\ 1 & \cdots & 2 & 3 \\ 4 & \cdots & . & 5 \\ 4 & \cdots & 5 & 6 \\ 7 & \cdots & . & 8 \\ 7 & \cdots & 8 & 9 \\ \vdots & \vdots & \vdots & \vdots \\ N-6 & \cdots & \cdot & N-5 \\ N-6 & \cdots & N-5 & N-4 \\ N-3 & \cdots & \cdot & N-2 \\ N-3 & \cdots & \cdot & N-1 \\ N-2 & \cdots & N-1 & N\end{array}$

In this case, every subset of each of the sets $\{1,2,3\}, \ldots,\{N-3, N-2$, $N-1, N\}$ is a union of rays, so every index set $J$ is a union of rays.

In either case Theorem 2 guarantees the existence of an independent collection of $N=\lfloor 3 d / 2\rfloor$ cubes.

\section{References}

Grünbaum, B. (1975). Venn diagrams and independent families of sets. Math. Mag. 48, 12-23.

Marczewski, E. (1947). Indépendance densembles et prolongements de mesures. Colloq. Math. 1, 122-132.

Naiman, D. Q., and Wynn, H. P. (1991a). Inclusion-exclusion bonferroni identities and inequalities for discrete tube-like problems via Euler characteristics. Ann. of Statist., to appear.

Naiman, D. Q., and Wynn, H. P. (1991b). Discrete Tube Theory in $R^{d}$. Unpublished manuscript.

Rényi, A., Rényi, C., and Surányi, J. (1951). Sur l'independence des domaines simples dans l'espace euclidien à $n$ dimensions. Colloq. Math. 2, 130-135.

Received March 21, 1991, and in revised form March 9, 1992. 